\title{
Integrated Case-based Neural Network Approach to Problem Solving.
}

\author{
B. Lees and J. Corchado
}

\author{
Department of Computing \& Information Systems \\ University of Paisley, Paisley PA1 2BE, Scotland UK
}

Tel: 01418483311

Fax: 01418483542

email lees-ci0@paisley.ac.uk

\begin{abstract}
A hybrid learning system is presented which combines the methodologies of Case Based Reasoning (CBR) and Artificial Neural Networks (ANN) in an agent-oriented architecture and which may learn from past experience and from feedback from the current state of its environment, in order to (i) forecast the state of the environment at some point in the future, and (ii) adapt its behaviour so as to deal with new unexpected situations. The work focuses on Adaptive Agents operating in complex environments in which constraints change in an unpredictable manner, an underlying domain model is not available, but where data on previous states of the environment are accessible. The CBR and ANN methods are used at different stages and with different goals during the learning and forecasting process. The approach is being tested in a practical situation with the aim of developing a methodology for predicting the physical structure, in three dimensions, around a sea going vessel from data acquired in situ and also from historical records.
\end{abstract}

\section{Introduction}

Recent advances in artificial intelligence research have shown that both symbolic Al and artificial neural networks (ANN) approaches to solving real-world problems have both their strengths and limitations. Current beliefs suggest that these approaches are complementary, rather than competitive, and that a methodology drawing on the strengths of both these approaches can result in a more powerful problem solving capability than could be accomplished by either technology on its own. In particular, connectionist approaches can represent the perceptual aspects of intelligence, whilst symbolic approaches are an effective means of modelling cognitive aspects. Future advances in Al problem solving systems will most likely require the use of hybrid systems which embody different problem solving paradigms. The results obtained in previous research (Lees et al., 1992; Lees and Irgens, 1991) confirms the need for such tools. The identification of the form and components of such hybrid systems, and their modes of interaction, is an important topic of current Al research. A desirable feature of such a system is to provide the means of enhancing software engineering systems. Indeed, it is the authors' belief that Al technology is likely to have a greater impact in industrial and commercial applications through the provision of software tools that provide the means of defining collections of intelligent agents that may be integrated into conventional software systems, than through large stand-alone complex Al systems.

The aim of the research outlined in this paper is to investigate the integration of multiple intelligent agents (based of different problem-solving paradigms) to provide support for cooperative problem solving. The problem solving methods of interest in this research are: artificial neural networks (ANN) and case-based reasoning (CBR). It is believed that a problem solving approach which integrates these methodologies will provide several advantages: 
(i) artificial neural networks provide a learning capability from detailed example situations, where explicit knowledge of the domain does not exist, or is not accessible;

(ii) case-based reasoning draws on a suitably indexed and catalogued set of previously encountered large-scale problem situations, for which whole or partial solutions have been obtained.

Until recently developments in symbolic $\mathrm{Al}$ and connectionist problem solving have progressed in parallel, but each of these approaches have their relative strengths and weaknesses. As a result, there is a growing research interest in the development of systems which incorporate both these methods. Harris and his colleagues at Brunel have investigated several possibilities (Harris et al, 1994). Issues relating to the integration of symbolic and ANN approaches have also been addressed by (Sun and Bookman, 1993; Towell and Shavlik, 1992). Research into the employment of artificial neural networks as a software engineering paradigm is reported by (Partridge, 1993). But such developments appear at present to be mainly limited to a combination of ANN and Knowledge Based Systems (KBS) methods. The possible integration of $\mathrm{CBR}$ with $\mathrm{ANN}$ and symbolic KBS, offers a further potential dimension in multi-paradigm Al problem solving.

After considering the characteristics of adaptive agents, the integration of symbolic and connectionist methods in a hybrid problem solving system is discussed. The practical application of the resulting prototype system in oceanographic data analysis is then outlined.

\section{Adaptive Agents}

The term agent is widely used by many people working in related areas and it is very difficult to produce a single universally accepted definition. Agents may be partitioned into static/mobile agents, deliberative/reactive agents, task/resource agents, for example. However the approach presented in this paper is based on that presented in (Wooldridge and Jennings, 1994) in which an agent is defined from the functional characteristics that it should possess: autonomy, social ability, reactivity and pro-activeness. In addition to having these properties, it is advantageous for an agent to be either conceptualised or implemented using concepts that are also applicable to humans. A discussion of various different attributes of agency and an attempt to define them formally, appears in (Goodwin, 1993).

An agent (Maes, 1995) is said to to be autonomous if it operates completely independently, i.e. if it is able to decide its elf how to relate its sensor data to motor commands in such a way that its goals are successfully achieved. An agent is adaptive if it is able to improve its performance over time, that is, if the agent becomes better at achieving its goals with accumulated experience. There is a continuum of ways in which an agent can be adaptive, from being able to adapt flexibly to short-term, smaller changes in the environment, to dealing with more significant and long-term (lasting) changes in the environment, that is, being able to change and improve its behaviour over time.

\section{Hybrid Problem Solving}

Very complex problems may not always be solved by the application of just one technique. Research in the area of hybrid system forms a relatively new field, in which an important aspect is the study of how to combine connectionist and symbolic Al techniques. In particular, it is beneficial to use a hybrid system when there is not enough (symbolic) knowledge about a system to use a symbolic algorithm or not enough data to train a neural network.

Case Based Reasoning and Artificial Neural Networks are complementary problem solving techniques (Corchado et al.,1997): CBR systems are able to reuse information from past experience: ANNs can generate adaptive structures using large data sets. Agents operating in complex environments, which change with time, can employ symbolic methods (e.g. CBR) to 
use past recorded knowledge, and other, connectionist, methods to act on data recorded in real time. An appealing feature of Artificial Neural Networks is that their structures and behaviour are modelled on the believed mechanisms of the human brain. Many complex tasks that a human being can perform with apparent ease are not so easily performed by computers using traditional algorithmic methods. These tasks are more appropriately carried out by computers whose structure and processing operations are similar to those found in the brain, as is the case for ANN (Rumelhart et al., 1996).

\section{Radial Basis Functions}

The particular neural network currently of interest in this research is the Radial Basis Function (Bishop, 1995). In a Radial Basis Function (RBF) the input layer is a receptor for the input data, whilst the hidden layer performs a non-linear transformation from the input space to the hidden layer space. The hidden neurons form a basis for the input vectors; the output neurons merely calculate a linear combination of the hidden neurons' outputs.

Activation is fed forward from the input layer to the hidden layer where a Basis Function, which is the Euclidean distance between the inputs and the centres of the basis function, is calculated. The weighted sum of the hidden neurons' activations is calculated at the single output neuron. RBFs are better at interpolating that at extrapolating. Where there is a region of the input space with little data, a RBF cannot be expected to approximate well, Furthermore, RBFs are less sensitive to the order in which data is presented to them than is the case with other ANN models, such as Multi Layer Perceptrons. However, Radial Basis Functions are useful in hybrid systems because of their fast learning capability.

\section{Integrating Neural and Case-Based Problem Solving}

The integration of ANNs with CBR, has been investigated by a relatively small number of researchers (Krovvidy et al., 1993; Kock, 1996). CBR techniques are effective in remembering specific cases, that may not be generalisable, whereas ANNs are effective in generalising information, extracting rules and clustering data. This integration can be achieved either through the division of tasks between CBR and ANN problem solvers, or through the design of an intelligent agent architecture combining ANN and CBR features. (Reategui et al., 1994) identifies four possible approaches for integrating CBR with other reasoning methods:

- central control. where the CBR and the other mechanisms are controlled by a central device;

- distributed control, where the control is divided between the all techniques;

- CBR dominant, where the control is biased towards the CBR component;

- CBR non dominant, where the control is biased towards the other component.

In most proposed methods of integrating ANN and CBR methods, the ANN is used for case matching and recovering of tasks, looking for patterns of similarity between cases.

CBR systems are particularly appropriate when rules from the knowledge domain are difficult to discern or the number and complexity of the rules is too large for the normal knowledge acquisition process; they have the potential to provide some of the human characteristics of problem solving that are difficult to simulate using the logical, analytical techniques of knowledge based systems and standard software technologies. ANNs are able to analyse large quantities of data to establish patterns and characteristics in situations where rules are not known and, in many cases, can make sense of incomplete or noisy data.

Once the agent or the human brain accumulates many cases or experiences about a particular problem it should be possible to generalise and extrapolate knowledge from those previous experiences. The human brain accomplishes this in a natural way using its (human) neural networks; similarly, an agent can achieve this using artificial neural networks, which 
can thus be used to forecast some state in the future and the CBR can be used to supervise their performance. Both the ANN and the CBR mechanism can be used as a means of forecasting and their outcomes can be compared. The ability of the ANN to generalise may produce better results than CBR when it has been trained with an appropriate data set. In this situation it would be possible to rely on the ANN and its generalising abilities. However, if the system is inserted in a dynamic environment, whose characteristics change in an unpredictable manner, an agent will require an adaptive mechanism capable of reacting to such changes. It is possible to endow a CBR mechanism with the ability to detect large changes in the environment. Normally, however, large changes will decrease the performance of the ANN, which is more effective when it has been trained with a significant amount of data representative of the whole possible data set. In such a situation CBR may be more effective than an ANN. As a result the ANN will be required to be retrained using the most recently retrieved cases, using the CBR mechanism.

An Adaptive Agent is required to modify the forecasting mechanism, depending on detected changes in the environment. Once the number of cases becomes very large, the agent will use cases that have recently been the most frequently retrieved, to train an ANN. Then both the CBR mechanism and the ANN may be used to obtain a forecast. When the ANN starts to produce less accurate outcomes than with the CBR method, its operation will be terminated and the agent will then create a new ANN to be trained with the most frequently retrieved cases held in the case base. Alternatively, the operation of an ANN may be frozen if the characteristics of the system change substantially. Such a frozen ANN can always be recalled to work in parallel with the CBR system and also with other ANNs. Thus the agent will have control over all the learning and forecasting mechanisms working at any point.

\section{Practical Application}

The multi-agent approach to prediction is being investigated in collaborative work with Plymouth Marine Laboratory, in which the aim is to develop a methodology for predicting the physical structure in three dimensions, around a sea going vessel from data acquired in real time, and also from historical records. A particular objective is to produce a prediction, in real time, of the distribution of sea temperature (and possibly other oceanographic parameters) surrounding the vessel at some point ahead of the vessel, which will be reached in the immediate future. This information may then be used to provide a forewarning of an impending oceanographic front, i.e. an edge between different water masses. The system will build on the methods and expertise previously developed at Plymouth Marine Laboratory, and, in particular, in previous collaborative work with the University of Paisley into the application of knowledge based methods for the analysis of oceanographic data (Lees et al., 1992).

\section{Data Pre-Processing}

The raw data (on sea surface temperature and other oceanographic parameters) is in the form of a discrete number of values of a parameter: in this case the temperature of the water mass. Values are sampled along a single horizontal dimension, thus forming a set of data points (a data row). The actual data used to test the ideas developed in this research were recorded during the cruise of a research vessel from the UK to the Falkland Islands.

This data must be pre-processed in order to eliminate noise, to enhance interesting features, to smooth stable areas and to transform the data set into a form which may be represented on an absolute scale. There are several techniques that can be applied to transform the original data row (Corchado, 1995) to reduce noise, sharpen data and aid in the detection of fronts. The approach adopted was to employ a Sobel Filter, the operation of which is based on the idea that local variations, corresponding to edge transitions, occur at a slower rate than those corresponding to noise. 


\section{Results and Discussion}

The approach presented in this paper combines the advantages of both connectionist and symbolic Al. So far, a simulation of the real system has been used for testing in the laboratory. Rather than sampling data with sensors, the simulator is fed with a sequence of pre-stored historic data values. The system in its present form will forecast the structure of the water $3 \mathrm{~km}$ ahead of an ongoing vessel using the data recorded in the past $7 \mathrm{~km}$. For the CBR system, a case consists of the output from a Sobel filter of a temperature row of sampled data over a $7 \mathrm{~km}$ stretch of water, together with values for the predicted temperature of the water over the next $3 \mathrm{~km}$. Once the system starts operating, the first requirement is to initialise the first case. After $10 \mathrm{~km}$ the first complete case will be stored, three $\mathrm{km}$ afterwards the next case will be recorded and so on. The CBR system is then ready to forecast. The forecasted results are compared with the known parameter values, from which an estimation of the reliability can be produced. After recording 500 cases, a RBF neural network is trained with all the cases so far recorded. The input to the ANN is the set of pre-processed data values from the Sobel filter; the centres of the RBF are chosen randomly from the input data and the output is the forecasted parameter values. If the performance of the ANN is worse than that of the CBR system over a distance greater than $10 \mathrm{~km}$, then that network is eliminated. Otherwise it is retained until its performance becomes worse than that of the CBR for a period bigger than $3 \mathrm{~km}$. At this point the process of prediction by the ANN is suspended, but the network is not discarded. A new NN is trained using the strongest 500 cases and the whole of the above cycle starts again.

Several CBR methods have been tested on the system to determine their effectiveness when used by autonomous agents, and, also, how they may improve their autonomy, with promising results so far. In future work it is intended to investigate the application of Fuzzy Sets and Neuro-fuzzy Algorithms to the multi-agent architecture. Neuro-fuzzy algorithms (Brown et al.,1995) aim to combine the learning abilities of artificial neural networks with the linguistic, rule-based knowledge of a fuzzy system. This provides a simple explanation facility for the otherwise opaque artificial neural networks, and allows domain experts to embed their knowledge inside the system. In addition, such systems can be primed with domain knowledge before learning commences, simplifying the task and improving the rate of convergence. It is thus aimed to provide the agents with greater autonomy by transforming the learning acquired by a Radial Basis Function network into an easy to understand Fuzzy set.

\section{Acknowledgement}

The authors wish to express their gratitude to their colleagues Professor Jim Aiken and Mr Nigel Rees at Plymouth Marine Laboratory, for their support in this collaborative research.

\section{References}

Bishop C. R., 1995. Neural Networks for Pattern Recognition, Carendon Press, Oxford.

Booker, L. Classifier systems that learn internal world models. Machine Learning Journal, 1(2).

Brown M., Abed Z. and Harris C.J.. Using the neurofuzzy approach to building dependable neural network systems. In Neural Networks - Producing Dependable Systems, pages 4.2.1--4.2.9. ERA, 1995

Corchado J. M. Application of Filters, Masks and Artificial Neural Networks to Oceanographic Frontal Warning, BSc Honours Dissertation, University of Paisley, 1995.

Corchado, J. M., Lees, B. and Rees, N. A Multi-agent system "test bed" for evaluating autonomous agents. (to appear) Procs. Agents-97, Los Angeles, 1997. 
Goodwin, R. Formalising properties of agents. Technical Report CMU-CS-93-159, School of Computer Science, Carnegie-Mellon University, May 1993.

Harris, T, et al., Neural networks, and expert systems: complementary technologies that can work together, Applications and Innovations in Expert Systems, edited by R Milne and A Montgomery, SGES Publications, 1994.

Kock, G. The neural network description language CONNECT,and its $\mathrm{C}_{++}$implementation. Technical report, GMD FIRST Berlin, Universitat Politecnica de Catalunya, August 1996.

Krovvidy, S. and Wee. W. C., Wastewater treatment systems from case-based reasoning. Machine Learning, 10:341-363, 1993.

Lees B and Irgens C. Knowledge based support for quality in engineering design, Procs. Eleventh International Conference on Expert Systems and their Applications, Avignon, May 1991, pp 257266.

Lees, B., Rees, N. and Aiken, J. Knowledge-based oceanographic data analysis, Procs. Expersys-92, editors: F Attia, A Flory, S Hashemi, G Gouarderes, J Marciano, IITT International Paris, October 1992, pp. 561-65.

Maes, P. (ed.) Designing Autonomous Agents, MIT/Elsevier,1993.

Partridge, D. Connectionism as a software engineering paradigm, Advanced Information Systems, vol 1(1), pp. 7-14, 1993.

Reategui, E. and Campbell, J. A. A classification system for credit card transactions. Procs. Second European Workshop on Case-Based Reasoning, pp. 167 174, November 1994.

Rumelhart, D. E and McClelland, J. L. Parallel Distributed Processing, Volume 1: Foundations, MIT Press, 1986.

Sun, R. and Bookman, L. How do symbols and networks fit together? Al Magazine, vol. 14(2), 20-23, 1993.

Towell, G. and Shavlik, J. W. Interpretation of artificial neural networks mapping knowledge-based neural networks into rules, In Advances in Neural Information Processing Systems, vol. 4, pp. 977-984, Morgan Kaufmann, 1992.

Wooldridge, M. and Jennings, N. R. Agent theories, architectures and languages: A Survey. Procs. ECAl-94 Workshop on Agent Theories, Architectures, and Languages, 1994. 